\title{
$C(X)$ IS NOT NECESSARILY A RETRACT OF $2^{X}$
}

JACK T. GOODYKOONTZ, JR.

\begin{abstract}
Let $X$ be a continuum and $2^{X}(C(X))$ denote the hyperspace of closed subsets (subcontinua) of $X$. An example is given of a continuum $X$ such that $C(X)$ is not a retract of $2^{X}$.
\end{abstract}

By a continuum $X$ we mean a compact connected metric space. $2^{X}$ denotes the hyperspace of closed subsets of $X$ with the finite (Vietoris) topology. $C(X)$ denotes the hyperspace of subcontinua of $X$ with the relative topology from $2^{X}$. Since $X$ is a continuum, each of $2^{X}$ and $C(X)$ is also a continuum (see [4]). If $Y \subset X$, then $Y$ is a retract of $X$ means that there exists a continuous function $r: X \rightarrow Y$ such that the restriction of $r$ to $Y$ is the identity on $Y$.

In this paper we obtain a partial answer to a question raised by Sam B: Nadler, Jr. In 1939 Wojdyslawski [7] proved that $C(X)$ is an absolute retract if and only if $X$ is locally connected. Hence $C(X)$ is a retract of $2^{X}$ when $X$ is locally connected. In Theorem 3.6 of [5] Nadler shows that $C(X)$ is always a continuous image of $2^{X}$. Following this result, in Question 3.7 of [5], Nadler asks "When is $C(X)$ a retract of $2^{X}$ ?" Nadler discusses his question further in [6] and considers the possibility that $C(X)$ is always a retract of $2^{X}$. Using an interesting approach with topological semilattices, Lawson [3] asserted that certain nonlocally connected continua admit a retraction from $2^{X}$ onto $C(X)$. However, there is an error in the proof of Proposition 4 in Lawson's paper (his function $\beta i$ is not a retraction from $2^{X}$ onto $C(X)$ ). ${ }^{1}$ Consequently, there is currently no known example of a nonlocally connected continuum $X$ such that $C(X)$ is a retract of $2^{X}$. It is the purpose of this paper to present an example of a continuum $X$ such that $C(X)$ is not a retract of $2^{X}$. The example is a slightly modified version of a subcontinuum of Example 3 of [1].

If $U_{1}, \ldots, U_{n}$ are open sets in $X$, then $N\left(U_{1}, \ldots, U_{n}\right)=\left\{A \in 2^{X} \mid A \subset\right.$ $\cup_{i=1}^{n} U_{i}$ and for each $\left.i=1, \ldots, n, A \cap U_{i} \neq \varnothing\right\}$. The collection of sets $N\left(U_{1}, \ldots, U_{n}\right)$ forms a base for the finite topology on $2^{X}$ (see [4]).

ExAmple. This example is a subspace of $E^{3}$ (Euclidean 3-space). If $x, y \in$ $E^{3}$ we let $[x, y]$ denote the line segment in $E^{3}$ joining $x$ and $y$. For each

Received by the editors January 7, 1977 and, in revised form, April 11, 1977.

AMS (MOS) subject classifications (1970). Primary 54B20; Secondary 54C15.

Key words and phrases. Continuum, hyperspace, retract.

'Carl Eberhart and Wiley Williams have observed that the example in this paper admits a semilattice structure which satisfies the hypothesis of Proposition 6 of [3] and, hence, provides a counterexample to Proposition 6. 
positive integer $n$ let

$$
A_{n}=[(0,1 / 2,1 / n),(0,1,0)]
$$

and

$$
\begin{aligned}
& B_{n}= {[(1,0,1 / n),(1 / n, 0,1 / n)] \cup[(1 / n, 0,1 / n),(0,1 / 2,1 / n)] } \\
& \cup[(0,1 / 2,1 / n),(-1 / n, 0,1 / n)] \cup[(-1 / n, 0,1 / n),(-1,0,1 / n)] .
\end{aligned}
$$

Let $X$ be the closure of $\cup_{n=1}^{\infty}\left(A_{n} \cup B_{n}\right)$ and let

$$
B=\operatorname{limit}_{n \rightarrow \infty} B_{n}=[(1,0,0),(-1,0,0)] \cup[(0,0,0),(0,1 / 2,0)] .
$$

Suppose that $r$ is a retraction from $2^{X}$ onto $C(X)$. Let $\dddot{y}^{\prime}=$ $N\left(V_{1}, \ldots, V_{m}\right) \cap C(X)$ be an open set such that $B \in \widetilde{Y}$ and such that each $V_{i}$ has diameter $<1 / 2$. Then $(0,1,0) \notin \cup \mathcal{V}$. Since $r(B)=B$ and $r$ is continuous at $B$, there exists an open set $\mathscr{U}=N\left(U_{1}, \ldots, U_{k}\right)$ such that $B \in \mathcal{Q l} \subset N\left(V_{1}, \ldots, V_{m}\right)$ and $r(\vartheta l) \subset \widetilde{T}$. Choose $\delta>0$ such that, for

$$
D=[(1,0,0),(-1,0,0)] \cup[(0,0,0),(0,1 / 2-\delta, 0)] \text {, }
$$

$D \in \mathcal{Q}$. Then there exists a sequence $D_{n} \rightarrow D$ such that for each $n, D_{n} \subset B_{n}$ and $D_{n} \in 2^{X}-C(X)$. Since $D_{n} \rightarrow D, r\left(D_{n}\right) \rightarrow r(D)=D$. Note that subcontinua of $X$ which are sufficiently close to $D$ must be contained in $B$. Hence there exists a positive integer $n_{1}$ such that for each $n \geqslant n_{1}, r\left(D_{n}\right) \subset B$. Since $D_{n} \rightarrow D \in \mathcal{Q}$ and $B_{n} \rightarrow B \in \mathcal{Q}$, there is a positive integer $n_{2}$ such that for each $n \geqslant n_{2}, D_{n} \in \mathcal{Q l}$ and $B_{n} \in \mathcal{Q l}$. It follows easily from the definition of the finite topology that if $n \geqslant n_{2}$ and $E \in 2^{X}$ such that $D_{n} \subset E \subset B_{n}$, then $E \in \mathcal{Q}$. Let $j=\max \left\{n_{1}, n_{2}\right\}$. By [2, Lemma 2.3] there is a segmment $\sigma$ : $[0,1] \rightarrow 2^{X}$ such that $\sigma(0)=D_{j}$ and $\sigma(1)=B_{j}$. Since $j \geqslant n_{2}$, it follows from [2, Definition 2.2] that $\sigma([0,1]) \subset$ Ql. Hence $r(\sigma([0,1]))$ is a subcontinuum of $C(X)$ contained in $\tau$. Furthermore, $r\left(D_{j}\right) \in r(\sigma([0,1]))$ and $B_{j}=r\left(B_{j}\right) \in$ $r(\sigma([0,1]))$. Let $A=\cup r(\sigma([0,1]))$. By [2, Lemma 1.2], $A$ is a subcontinuum of $X$. Since $B_{j} \subset A, r\left(D_{j}\right) \subset A$, and $r\left(D_{j}\right) \subset B$, it is easy to see that $(0,1,0)$ $\in A$. However, since $r(\sigma([0,1])) \subset \widetilde{\top}, A \subset \cup \widetilde{\top}$, and since $(0,1,0) \notin \cup \widetilde{\top}$. $(0,1,0) \notin A$. We have a contradiction. Hence $C(X)$ is not a retract of $2^{X}$.

\section{REFERENCES}

1. J. T. Goodykoontz, Jr., More on connectedness im kleinen and local connectedness in $C(X)$, Proc. Amer. Math. Soc. 65 (1977), 357-364.

2. J. L. Kelley, Hyperspaces of a continuum, Trans. Amer. Math. Soc. 52 (1942), 23-36.

3. J. D. Lawson, Applications of topological algebra to hyperspace problems, (Proc. Memphis State Univ. Topology Conf.), Lecture Notes in Pure and Appl. Math., no. 24, Dekker, New York, 1976, pp. 201-206.

4. E. Michael, Topologies on spaces of subsets, Trans. Amer. Math. Soc. 71 (1951), 152-182.

5. S. B. Nadler, Jr., Some problems concerning hyperspaces, Lecture Notes in Math., vol. 375, Springer-Verlag, New York, 1974, pp. 190-197.

6 . A characterization of locally connected continua by hyperspace retractions, Proc. Amer. Math. Soc. 67 (1977), $167-176$.

7. M. Wojdyslawski, Retractes absolus et hyperespaces des continus, Fund. Math. 32 (1939), $184-192$.

Department of Mathematics, West Virginia University, Morgantown, West Virginia 